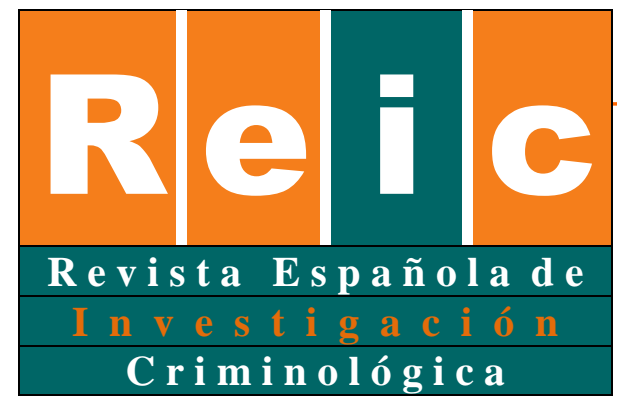

Rovira

\title{
El estigma de los antecedentes penales en el mundo laboral. Un estudio experimental
}

\section{The stigma of criminal records in the labour market. An experimental study}

Recibido el 19 abril 2017/Publicado el 29 diciembre 2017

\author{
Martí Rovira ${ }^{1}$ \\ Universitat Pompeu Fabra
}

\begin{abstract}
RESUMEN
Este trabajo se dirige a determinar si se debe considerar el efecto del estigma de los antecedentes penales para explicar la precariedad laboral de las personas con una condena en España. Los estudios previos no podían separar este efecto de otras posibles explicaciones como, por ejemplo, la prevalencia de déficits formativos o la ruptura de redes sociales por la condena. En base a Pager (2007), en este estudio se ha adoptado la metodología experimental de los Correspondence Tests, en la que se envían Currículums Vitae falsos equivalentes, excepto en la mención de un paso por prisión, a ofertas de trabajo reales, y se observa la diferente tasa de respuesta a cada perfil. Los resultados muestran que al menos parte de las personas con antecedentes penales reciben un trato desfavorable por el efecto del estigma.
\end{abstract}

Palabras clave: Estigma, antecedentes penales, prisión, discriminación, empleo.

\section{ABSTRACT}

\footnotetext{
${ }^{1}$ La correspondencia debe dirigirse a: Martí Rovira. Departamento de Derecho. Universitat Pompeu Fabra. Edificio Roger de Llúria (Campus de la Ciutadella). C/ Ramon Trias Fargas, 25-27. 08005 Barcelona. marti.rovira@upf.edu / m.rovira.sopena@gmail.com
} 
This research is directed to establish if the stigma of a criminal record should be considered in understanding the worse conditions in the labour market of people with convictions in Spain. Previous studies were unable to separate this effect from other explanations as low levels of education or the breakdown of networks because of the conviction. Following Pager (2007), this study adopts the experimental methodology of Correspondence Test, on which matched fake resumes, that only differ in a mention of a prison stay, are sent to real job offers, to test if there is a difference in the responses between the resumes. The results show that in Spain, at least some part of the people with criminal records appear to be discriminated in the labour market because of stigma.

Keywords: Stigma, criminal records, prison, discrimination, employment.

\section{Introducción}

Las personas que han pasado por el sistema penal tienden a encontrarse en una situación de precariedad en el mundo laboral. La investigación internacional ha observado de forma consistente una asociación entre una condena y unos salarios relativamente bajos y una gran inestabilidad laboral (Apel \& Sweeten, 2010; van der Geest, Bijleveld, Blokland, \& Nagin, 2016; Visher \& Kachnowsi, 2007). Esta asociación también se ha constatado en España (Alós-Moner et al., 2011; Cid \& Martí, 2012; Ríos \& Cabrera, 1998).

La asociación entre el paso por el sistema penal y la precariedad laboral es preocupante. La gran mayoría de teorías criminológicas plantean que el empleo, especialmente el de calidad (Crutchfield, 2014; Uggen \& Staff, 2001), es un factor fundamental para evitar la reincidencia (Jacobs \& Larrauri, 2011; Uggen \& Staff, 2001). Se ha afirmado que el empleo proporciona a una persona ingresos para conseguir el nivel de vida esperado (Merton, 1938); promueve valores, actitudes y comportamientos convencionales (Sutherland \& Cressey, 1978); fortalece contactos pro-sociales (Sampson \& Laub, 1993; Uggen \& Staff, 2001) y facilita la construcción de una "narrativa optimista” y una imagen positiva de sí misma que favorece su rehabilitación (Cid \& Martí, 2012; Maruna, 2011).

En los últimos años ha vuelto a adquirir relevancia la posibilidad de que la precariedad laboral de las personas condenadas se explique, en parte, por el estigma de 
los antecedentes penales (Bushway, 1998; Nagin \& Waldfogel, 1995; Pager, 2007; Uggen, Manza, \& Thompson, 2006; van der Geest et al., 2016; Verbruggen, 2016; Western, 2002). Por un lado, se ha señalado la existencia de diferentes leyes que dificultan la inserción laboral de las personas que han sido condenadas con anterioridad (Mauer \& Chesney Lind, 2003). Por otro lado, se ha destacado que el paso por el sistema penal puede actuar como un atributo que genera rechazo en el mundo laboral (aspecto señalado por el mismo Goffman, 1990), de forma que los responsables de selección de personal discriminen $^{2}$ a las personas con antecedentes penales (LeBel, 2008; Pager, 2007). Este efecto se podría haber incrementado recientemente en el contexto de un crecimiento de las actitudes relacionadas con la "cultura del control" (Cesaroni \& Doob, 2003; Díez Ripollés, 2004; Garland, 2001).

El efecto del estigma es preocupante en la medida en que supondría una pena "añadida" a la establecida en la sentencia, un castigo "invisible" fuera del control del sistema penal (Demleitner, 1999; Mauer \& Chesney Lind, 2003). Desde un punto de vista criminológico, se ha señalado que el estigma de la condena podría ser un obstáculo para la reinserción (por ejemplo, ver Denver, Siwach, \& Bushway, 2017), llevando a una rueda entre precariedad laboral, delincuencia, paso por el sistema penal y más precariedad laboral (Western, 2002). Ademas, los efectos del estigma pueden afectar negativamente no sólo al individuo, sino también a su familia y a su comunidad (Clear, 2008).

\subsection{La necesidad de separar el efecto del estigma de otras explicaciones}

No es sencillo realizar una investigación sobre el efecto del estigma. Los estudios previos han encontrado el problema de separar este efecto de otras potenciales explicaciones de la asociación entre el paso por el sistema penal y la precariedad laboral (Pager, 2007; Western, 2002).

En primer lugar, según las teorías del efecto selección no existe una relación de causalidad entre la condena y la precariedad laboral, sino una correlación entre estos dos factores: las mismas personas que son condenadas también muestran, con frecuencia, una serie de características negativas para encontrar empleo (Grogger, 1995; Loeffler, 2013; Ramakers, van Wilsem, \& Apel, 2012), ya que ambas variables se encontrarían

\footnotetext{
${ }^{2}$ En este trabajo se utilizará el término discriminar en su acepción sociológica, entendiendo este concepto como "seleccionar excluyendo" (Real Academia Española, 2016b).
} 
relacionadas, de forma independiente, con otros factores. En este sentido, Metcalf, Anderson y Rolfe (2001) señalan que la población condenada muestra, con mayor frecuencia que la población general, problemas de salud mental y de drogadicción, falta de vivienda, déficits en habilidades sociales, escasa cualificación, alto historial de desempleo, y una baja autoestima. Estos factores influirían, por una parte, en una mayor probabilidad de cometer un delito y ser condenado, e, independientemente, en una mayor precariedad laboral.

En segundo lugar, las teorías del efecto pena mencionan que el cumplimiento de las condiciones impuestas en la sentencia puede implicar un coste de oportunidad en términos monetarios y de tiempo, que puede impedir invertir en "capital humano", como la formación (Lott, 1990) o en mantener unas redes sociales proclives al empleo (Western, 2002). Además, algunos autores sugieren que la condena puede transformar al individuo, desmotivándolo para encontrar empleo y empobreciendo sus competencias (Apel \& Sweeten, 2010; Miguélez, Alós-Moner, Martín, \& Gibert, 2006).

Es importante delimitar la existencia de cada una de estas explicaciones ya que cada una apunta a una política pública diferente. Intuitivamente, las políticas relacionadas con el efecto selección señalan la necesidad de proveer a la población condenada de formación o de tratamiento psicológico en prisión para paliar estos déficits que frecuentemente presenta. Por otro lado, las teorías del efecto pena establecen la necesidad de que los reclusos trabajen en prisión, para evitar efectos desocializadores, y destacan la importancia de que mantengan un contacto con el exterior, para promover el mantenimiento de sus redes sociales. En otra línea, las políticas relacionadas con el efecto del estigma priorizan la realización de campañas para disminuir los prejuicios hacia las personas condenadas o, alternativamente, dificultar el conocimiento de esta condición a los responsables de selección de personal.

\subsection{Las posibilidades de las metodologías de los Correspondence Testsy los Audit Tests para aislar el efecto del estigma}

La aparición de las metodologías de los Correspondence Tests y los Audit Tests ha supuesto un gran avance en el estudio del efecto del estigma de los antecedentes penales en el mercado laboral. En la investigación más destacada, Pager (2007), mediante un Audit Test, envió a dos candidatos a solicitar las mismas ofertas de empleo. Los 
Currículums Vitae (en adelante CV o currículos) que presentaban los candidatos eran equivalentes, sólo se diferenciaban en que en uno de ellos se hacía constar que la persona tenía un antecedente penal. Mediante este experimento Pager observó que, de media, los antecedentes penales reducían la probabilidad de encontrar empleo en un 50\% de los $\operatorname{casos}^{3}$. De esta forma, la autora consiguió demostrar, de una forma intuitiva a la vez que científicamente rigurosa, la existencia del efecto del estigma, independientemente de otros efectos. Posteriormente otras investigaciones han reproducido este experimento a través de un Correspondence Test, una metodología prácticamente idéntica, pero en la que se mandan los CV a través de Internet, fax o correo postal (Decker, Ortiz, Spohn, \& Hedberg, 2015; Galgano, 2009) $)^{4}$.

Las metodologías de los Correspondence y los Audit Tests permiten superar los problemas de anteriores aproximaciones. Por un lado, a diferencia de las encuestas (ver, entre otros, Holzer, Raphael, \& Stoll, 2007) o de las entrevistas en profundidad (ver Backman, 2012), estas técnicas permiten captar el comportamiento real de los responsables de selección de personal. Por otro lado, estas metodologías permiten controlar el resto de variables intervinientes en esta relación, a diferencia de los estudios realizados mediante un seguimiento longitudinal (ver Ramakers et al., 2012) o por la unión de bases de datos de empleo y del sistema de justicia penal (ver Lott, 1990; van der Geest et al., 2016; Verbruggen, 2016). De esta forma, estas metodologías permiten asegurar que las únicas diferencias consisten en la variable de interés, aspecto que permite descartar totalmente la existencia del efecto selección o del efecto pena.

Sin embargo, es importante señalar que estas metodologías no permiten determinar el grado de discriminación o de precariedad de las personas con antecedentes penales en el mercado laboral (Heckman, 1998), sino sólo testar que este mecanismo está presente. Se conoce que la mayor parte de empleos se encuentran a través de contactos personales (Granovetter, 1995; para España ver Rieucau, 2008), aspecto que puede mitigar el efecto del estigma y que queda fuera de las posibilidades de estudio de los Correspondence y los Audit Tests. Además las personas condenadas también pueden

\footnotetext{
${ }^{3}$ Pager (2007) envió a solicitar trabajos a un par de jóvenes caucásicos y a un par de jóvenes afroamericanos. En cada par de solicitantes, aleatoriamente uno disponía de antecedentes penales y otro no. No se detectaron diferencias en el efecto del estigma entre estos dos grupos.

${ }^{4}$ Para una descripción de los resultados de todos los Audit y Correspondence Tests sobre esta cuestión ver Rovira (2016).
} 
ocultar esta condición en los procesos de selección de personal (Harding, 2003), por lo que los empleadores a menudo desconocen su situación penal. En este sentido, las metodologías de los Correspondence y los Audit Tests sólo permiten determinar si los responsables de selección de personal tratan desfavorablemente a las personas que muestran una señal del paso por el sistema penal en su currículo. Para cuantificar el grado de discriminación o precariedad laboral de la población condenada son más apropiadas las investigaciones mediante bases de datos administrativas o estudios longitudinales con comparaciones entre personas con antecedentes penales y un grupo de control equivalente.

\subsection{Objetivo y relevancia del estudio}

Centrándonos en España, la investigación previa ya había sugerido que el estigma de los antecedentes penales ${ }^{5}$ puede tener un efecto negativo en la inserción laboral de las personas condenadas (Alós-Moner et al., 2011; Bueno Arús, 2006; Grosso Galván, 1984; Gutiérrez-Maldonado \& Sintas, 1995; Ríos \& Cabrera, 1998; Roldán, 2010). En este sentido Larrauri y Jacobs (2011) demuestran que existen una serie de leyes que impiden trabajar a las personas con antecedentes penales en numerosas profesiones y sugieren la posibilidad de que el certificado de antecedentes penales se esté requiriendo también en otro tipo de empleos.

Sin embargo, la investigación empírica desarrollada en España no ha permitido constatar que estas personas sean efectivamente discriminadas en el mercado laboral por el efecto del estigma. Hasta el momento el comportamiento de los responsables de selección de personal ante una señal de que el candidato haya pasado por el sistema penal sólo se ha abordado de forma indirecta a través de las apreciaciones de los propios condenados (Alós-Moner et al., 2011; Ríos \& Cabrera, 1998; Roldán, 2010) o mediante investigaciones de carácter exploratorio, con una muestra insuficiente para poder extrapolar los resultados (Gutiérrez-Maldonado \& Sintas, 1995). No se ha realizado ningún Audit Test ni Correspondence Test, por lo que, en base a los motivos ya señalados,

\footnotetext{
${ }^{5}$ En este artículo se utiliza el concepto de "antecedentes penales", para designar la "circunstancia consistente en haber sido alguien anteriormente condenado u objeto de persecución penal" (Real Academia Española, 2016a). Esta acepción es más amplia que el uso jurídico de este concepto, limitado a los registros de condenas del Ministerio de Justicia.
} 
no se ha podido determinar si existe efectivamente un trato desfavorable en el mercado laboral hacia las personas con antecedentes penales a causa del estigma.

Una investigación sobre la existencia de este fenómeno es particularmente relevante en la actualidad. Desde la entrada en vigor de la Ley 26/2015, de 28 de julio, de Protección a la Infancia y a la Adolescencia, los responsables de selección de personal deben requerir la presentación de un certificado de carecer de condenas de carácter sexual a las personas que trabajan en empleos que supongan un contacto habitual con menores. Esta modificación legal ha situado en el debate público la posibilidad de requerir información de este tipo en los procesos de selección de personal (Rovira, 2016). Thomas (2007) y Backman (2012) han sugerido que tras la aprobación de una ley de este tipo se incrementó la petición de información sobre condenas en el mercado laboral en el Reino Unido y Suecia respectivamente.

En este contexto, en este artículo se presentan los resultados de un estudio desarrollado en España para determinar la existencia del efecto del estigma de los antecedentes penales en la probabilidad de encontrar empleo, realizado mediante la metodología experimental de los Correspondence Tests. A continuación, se explica con detalle el diseño de investigación, por la novedad que comporta un estudio de estas características en España, y se resumen los primeros resultados.

\section{Metodología}

\subsection{Pregunta de investigación e hipótesis}

Esta investigación se orientó a responder a la pregunta: ¿Las personas con antecedentes penales reciben un trato desfavorable en los procesos de selección de personal?

Se estableció como hipótesis que los responsables de selección de personal tienen prejuicios contra las personas con antecedentes penales. En concreto, en la línea de otros Correspondence y Audit Tests (Pager, 2007), se dispuso que existiría discriminación debido a los antecedentes penales si el candidato con la "señal" obtuviera una probabilidad de respuesta positiva significativamente menor que el candidato sin ella.

\subsection{Consideraciones éticas}


Un Correspondence Test precisa atender especialmente a consideraciones éticas ante la falta de consentimiento para la participación en el estudio de los responsables de selección de personal. Los códigos éticos sobre investigación social permiten los estudios sin el consentimiento expreso de los sujetos de investigación si se cumplen una serie de condiciones: 1) que su valor científico esté justificado; 2) que no sea posible obtener esta información por otros medios; y 3) que los prejuicios para los sujetos de investigación estén reducidos al mínimo (Riach \& Rich, 2004).

Esta investigación ha buscado cumplir con estos requisitos. En primer lugar, se consideró razonable utilizar esta metodología ya que en España no hay estudios concluyentes sobre esta cuestión. En segundo lugar, como ya se ha justificado, se estableció que la metodología de los Correspondence Tests es la única que permite una aproximación al estudio del estigma en el mercado laboral. Por último, en la línea de las investigaciones precedentes (Pager, 2007), para minimizar los inconvenientes de los sujetos de investigación, se aseguró la confidencialidad de las empresas y del portal de ofertas de empleo utilizado. A la vez, se creó un protocolo para declinar la oferta lo más rápido posible, de tal forma que el experimento comportara mínimas molestias a los empleadores.

\subsection{Diseño de investigación}

En el marco de esta investigación se enviaron cuatro CV de candidatos ficticios a 601 ofertas de trabajo reales. Los cuatro currículos se diferenciaban atendiendo a dos elementos. Por un lado, dos de los CV mostraban una señal de un paso por el sistema de justicia penal, mientras que los otros dos no disponían de aquella marca o señal. Por otro lado, para cada grupo, los CV se diferenciaban entre sí por un diferente nivel de habilidades -medio o alto- de los candidatos. En el resto de condiciones los currículos eran exactamente equivalentes, con el objetivo de mantener el criterio de ceteris paribus, pero no iguales, para evitar la detección por parte de los responsables de selección de personal. A continuación se describe el proceso de creación de los $\mathrm{CV}$, en relación a estos tres elementos.

\section{a) Señales para mostrar el estigma del paso por el sistema penal}


Se introdujeron dos señales en los CV para mostrar la existencia de un antecedente penal. En concreto, en los currículos con señal del antecedente penal se introdujeron dos marcas: un curso relacionado con la oferta de trabajo, explícitamente realizado en prisión, y una recomendación de un técnico de inserción laboral del CIRE, el Centre de Iniciatives per a la Reinserció de la Generalitat de Catalunya, organismo público que se dedica a ofrecer apoyo para encontrar empleo a las personas que han terminado una condena. Se escogieron estas marcas, inspiradas en la investigación de Pager (2007) realizada en Estados Unidos, porque se consideraron la única forma realista de introducir en el currículo señales evidentes de que la persona había pasado por el sistema penal. Entrevistas realizadas a personas con antecedentes penales en otra investigación señalaron su convencimiento de que, en base a su experiencia, este tipo de señales eran consideradas por los responsables de selección de personal (Rovira, 2016) ${ }^{6}$.

Para asegurar el criterio de Ceteris Paribus, se introdujeron señales equivalentes en los $\mathrm{CV}$ de los candidatos de control. En contraste con las señales del otro grupo, en estos CV se añadió un curso similar al anterior relacionado con la oferta de trabajo, realizado a través del Servei d'Ocupació de Catalunya, el servicio público catalán encargado de la inserción laboral de la población desempleada, y una recomendación del último empresario que había empleado al candidato.

En los cuatro CV se situó un período de un año y medio seguido de inactividad laboral. En el caso de los candidatos con antecedentes penales, este período tendría que ser interpretado como un período en el que el candidato estuvo en prisión. En Cataluña, las personas excarceladas pasan, de media, menos de un año y medio en un centro penitenciario (Departament de Justícia. Generalitat de Cataluña, 2015b). En los candidatos sin marca, este período debía ser interpretado como un período de inactividad laboral.

\section{b) Señales para mostrar un diferente nivel de habilidades}

\footnotetext{
${ }^{6}$ En esta investigación se señaló que más allá de los certificados de antecedentes penales, las personas condenadas deben lidiar con otro tipo de señales que delatan su condición, como, por ejemplo, certificados de formación realizados dentro del sistema penal, información contenida en Internet o información transmitida por una red de conocidos común.
} 
En base a los últimos desarrollos en la metodología de los Correspondence Tests (Bertrand \& Mullainathan, 2004) se optó por diferenciar las habilidades de los candidatos. En concreto, para cada par de CV se envió un perfil medio y un perfil alto de habilidades. Se decidió no incluir ningún perfil bajo debido al contexto de crisis económica en el momento de realizar la investigación ${ }^{7}$. Se asumió que si el CV se correspondía con el de un joven sin formación acreditada de ningún tipo, producto de un abandono escolar temprano y sin experiencia laboral, la probabilidad de respuesta a solicitudes de candidatos de estas características sería muy baja.

Los dos perfiles de habilidades creados se diferenciaron atendiendo al nivel educativo y a la experiencia laboral. En este estudio, el perfil de habilidades calificado como "medio" corresponde al perfil mayoritario de los ex-presos mostrado en la investigación de Alós-Moner et al. (2011) y se ha caracterizado como el de una persona que dispone del certificado de Educación Secundaria Obligatoria (ESO) y dos años de experiencia laboral adquirida en cinco empleos distintos. El perfil de habilidades considerado como "alto" corresponde a una persona que ha estado siempre estudiando o trabajando, excepto el período "vacío", que, en el caso del CV con antecedentes penales, marcaría el paso por prisión. En concreto, se refiere a candidatos con un grado medio o superior de formación profesional relacionado con la profesión, y cinco años y medio de experiencia laboral, a partir de tres experiencias distintas.

\section{c) Señales equivalentes para el resto de características}

Con los cuatro modelos antes comentados de $\mathrm{CV}$, se crearon currículos para diferentes ocupaciones, de tal forma que los resultados fueran lo más representativos posibles del mercado laboral al que acceden los ex-presos (ver Alós-Moner et al., 2011). En concreto, se definieron CV para solicitar empleo de hasta 12 perfiles ocupacionales diferentes, de diversos sectores: atención al cliente, auxiliar del sector sociosanitario, carretillero, cocinero, dependiente, electromecánico, mánager, manipulador, matricero, mozo, transportista y vendedor. Los perfiles ocupacionales fueron escogidos en base a que presentaran diferencias competenciales relativas al contacto con el cliente, la complejidad de las tareas requeridas y el nivel de decisión. Para cada uno de estos sectores, se crearon

\footnotetext{
${ }^{7}$ En el segundo trimestre de 2012, la tasa de desempleo de los jóvenes entre 20 y 24 años en España era del $44.78 \%$ (Instituto Nacional de Estadística, 2015).
} 
CV con experiencia y formación en relación al sector, pero perfectamente equivalentes entre sí.

Para mantener el criterio de Ceteris Paribus, en el resto de características sociodemográficas o relacionadas con la productividad, los candidatos eran exactamente equivalentes. En primer lugar, todos ellos eran hombres jóvenes. Los hombres jóvenes son un caso paradigmático para evaluar los efectos del estigma de una condena. En el 2010 el $91.3 \%$ de las personas que salieron de un centro penitenciario en Catalunya fueron hombres (Departament de Justícia. Generalitat de Cataluña, 2015a). Por otro lado, se tomó la decisión de centrarse en jóvenes porque el estigma de una condena puede tener mayores efectos en la trayectoria laboral de este colectivo. Por este motivo, todas las fechas de nacimiento en los currículos eran de $1986^{8}$ y, por lo tanto, los candidatos tenían entre 26 y 27 años en el momento de buscar empleo. También se decidió que todos ellos mostraran señales de tener ascendencia española. En España la inmigración extranjera es un fenómeno nuevo y sería muy difícil hacer equivalencias de este grupo con el nivel formativo y la experiencia laboral de la población autóctona. Para destacar que la población era de origen autóctono se generaron los nombres y apellidos entre los 50 más frecuentes en España a partir de la lista facilitada por el INE para 2012 (Instituto Nacional de Estadística, 2013) ${ }^{9}$. Por otro lado, se utilizaron sujetos con apariencia caucásica en todas las fotografías. Finalmente, todos los candidatos ficticios mostraban unas habilidades medias en informática, disponían de carnet de conducir y tenían coche.

Finalmente, para evitar sospechas, los $\mathrm{CV}$ incorporaban diferencias en los nombres y apellidos, las fechas de nacimiento, los números de identificación personal (DNI), las direcciones de correo electrónico, los números de teléfono y los nombres de empresas y escuelas ${ }^{10}$. En algunos casos también se cambiaron algunos datos dentro de la sección del CV “Otros conocimientos” porque, de otro modo, los currículos resultaban

\footnotetext{
${ }^{8}$ La edad, entre 26-27 años, se escogió porque durante la fase piloto se planteó ir a recoger datos de otras fases del proceso de selección de personal. En un primer momento, el autor y otro investigador, muy parecidos entre sí, fueron a 7 entrevistas de trabajo diferentes que habían recibido una respuesta después de enviar el CV, siguiendo la investigación de Pager (Pager, 2007). En este contexto, se estableció esta edad ya que era aproximadamente la de los sujetos de investigación. Finalmente, esta parte se suprimió por ser demasiado costosa y suscitar dudas éticas, ya que se hacía perder mucho tiempo a los sujetos de investigación.

${ }^{9}$ De esta lista se suprimió el nombre Mohamed.

${ }^{10}$ Todos los nombres de escuelas y empresas eran ficticios, pero inspirados en escuelas y empresas reales.
} 
demasiado similares. Las fotografías también eran diferentes. Un diseñador gráfico creó dieciséis fotografías a partir de la combinación de elementos de cuatro fotografías del propio autor y de cinco personas más. Se modificaba la fotografía de cada currículo cada cuatro semanas de forma aleatoria ${ }^{11}$.

\subsection{Muestra}

Se enviaron los cuatro CV a 601 ofertas de trabajo. Los envíos se realizaron en dos períodos. En una fase inicial, del 17 de mayo al 30 de junio de 2012, se presentaron currículos a 246 ofertas de trabajo, un número muy similar a la muestra de 250 casos que utilizó Pager (2007) para su investigación. Ante la baja tasa de respuesta, en una fase posterior, del 10 de octubre de 2012 al 2 de febrero de 2013, se enviaron currículos a 355 nuevas ofertas de trabajo.

Se enviaron $\mathrm{CV}$ a todas aquellas ofertas de trabajo para personas residentes en la provincia de Barcelona durante ese período que requerían niveles bajos o medios de formación y escasa experiencia laboral en alguna de las ocupaciones de los perfiles descritos anteriormente. Se utilizó un portal de ofertas de trabajo en Internet que contenía empleos de todos los sectores. Como sólo se disponía de 16 fotografías y 16 líneas telefónicas y se enviaban cuatro currículos a cada oferta, sólo se podían gestionar ofertas de cuatro perfiles ocupacionales a la vez. El orden de solicitud del tipo de CV y la profesión por la que se empezaba variaba diariamente.

\subsection{Respuestas}

Se registró si para cada tipo de CV se recibía una llamada telefónica o un correo electrónico invitando al candidato a proseguir el proceso de selección. En concreto, se recibieron 192 respuestas a las solicitudes de empleo, de las cuales 149 se pudieron relacionar con el proyecto $^{12}$. En todas ellas se invitaba a la persona a proseguir el proceso

\footnotetext{
11 Un análisis posterior confirmó que no se encontraron diferencias relevantes en el tratamiento desfavorable según la fotografía.

${ }^{12}$ En 32 ocasiones se observó que la llamada no estaba relacionada con el proyecto. El número de línea había pertenecido a otras personas anteriormente y las llamadas se dirigían a ellos. En los 11 casos restantes no se logró identificar quién había llamado, por lo que no se pudo vincular a ninguna oferta. Se cree que la mayor parte eran llamadas no vinculadas al proyecto.
} 
de selección. Se registraron aquellas respuestas que se recibieron en las cuatro semanas posteriores al envío de la solicitud de empleo.

\section{Resultados}

La Figura 1 muestra los primeros resultados de la investigación. En su conjunto, los falsos candidatos con antecedentes penales han recibido una menor proporción de respuestas para proseguir el proceso de selección de personal que aquellos sin la marca, para los dos niveles de habilidades.

En concreto, en el nivel de habilidades alto, los CV con antecedentes penales recibieron una respuesta positiva en el $6.49 \%$ de los casos mientras que aquellos sin la señal recibieron una respuesta positiva en el $10.15 \%$ de los casos. Esto indica que para el grupo con un nivel de habilidades y formación alto, el hecho de mostrar el paso por el sistema penal reduce las probabilidades de éxito en los procesos de selección de personal en un $36 \%$.

En el nivel de habilidades medio, los CV con una señal de un antecedente penal recibieron una respuesta para proseguir el proceso de selección de personal en el $3.49 \%$ de los casos mientras que aquellos sin esta marca recibieron una respuesta positiva en $4.66 \%$ de las solicitudes de empleo. Esto señala que para el grupo de habilidades y formación media una señal de disponer de antecedentes penales reduce las probabilidades de empleo en un $25.1 \%$.

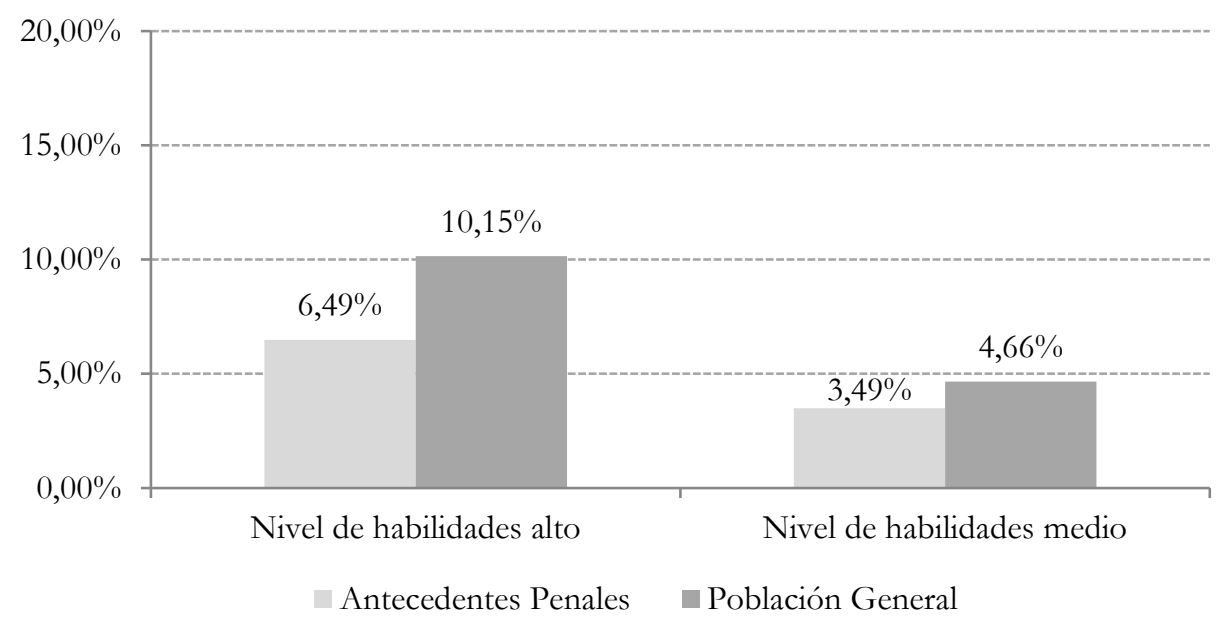

Figura 1: Porcentajes de llamada según nivel de habilidades y la señal de los antecedentes penales 
Para calcular si las diferencias en la probabilidad de respuesta para ambos perfiles de habilidades son significativas se ha utilizado el Test McNemar. El Test McNemar es un test estadístico adecuado para realizar pruebas de contraste para datos nominales de muestras pareadas (Vuolo, Uggen, \& Lageson, 2016). Este test contrasta la hipótesis de igualdad en la probabilidad marginal de ser contactado. El nivel de significación se ha establecido en el 0,05, por lo que se aceptará la hipótesis alternativa: existen diferencias en la probabilidad de llamada entre los currículos con antecedentes penales y aquellos otros sin antecedentes penales cuando el $P$-valor sea inferior a 0.05 .

La Tabla 1 muestra que los resultados del Test McNemar son significativos para los candidatos de perfil de habilidades alto $(P$-valor $<0.05)$. Se observa que en un $5.3 \%$ de los casos se contactó sólo al currículo sin la señal de los antecedentes penales, mientras que sólo se contactó al currículo con una señal del paso por prisión en un 1.7\% de los casos. El Test McNemar confirma que esta diferencia es estadísticamente significativa con una probabilidad de 0,001 . 


\section{Tabla 1.}

Resultados del Test McNemar según nivel de habilidades de los candidatos

\begin{tabular}{|c|c|c|c|c|c|c|c|}
\hline & \multirow{2}{*}{$\begin{array}{c}\mathrm{N}^{\mathrm{o}} \\
\text { ofertas }\end{array}$} & \multirow{2}{*}{\begin{tabular}{c}
\multicolumn{1}{c}{ Sin } \\
respuesta \\
en ambos \\
perfiles
\end{tabular}} & \multirow{2}{*}{$\begin{array}{c}\text { Respuesta } \\
\text { a ambos } \\
\text { perfiles }\end{array}$} & \multirow{2}{*}{$\begin{array}{c}\text { Respuesta sólo } \\
\text { al perfil con } \\
\text { antecedentes } \\
\text { penales }\end{array}$} & \multirow{2}{*}{$\begin{array}{l}\text { Respuesta sólo } \\
\text { al perfil sin } \\
\text { antecedentes } \\
\text { penales }\end{array}$} & \multicolumn{2}{|c|}{ McNemar Test } \\
\hline & & & & & & $\mathrm{X}^{2}$ & $\begin{array}{c}P- \\
\text { valor }\end{array}$ \\
\hline $\begin{array}{l}\text { Perfil } \\
\text { Alto }\end{array}$ & 601 & $\begin{array}{c}530 \\
(88.2 \%)\end{array}$ & $\begin{array}{c}29 \\
(4.8 \%)\end{array}$ & $\begin{array}{c}10 \\
(1.7 \%)\end{array}$ & $\begin{array}{c}32 \\
(5.3 \%)\end{array}$ & $10.500 * * *$ & 0.001 \\
\hline $\begin{array}{l}\text { Perfil } \\
\text { Medio }\end{array}$ & 601 & $\begin{array}{c}566 \\
(94.2 \%)\end{array}$ & $\begin{array}{c}14 \\
(2.3 \%)\end{array}$ & $\begin{array}{c}7 \\
(1.2 \%)\end{array}$ & $\begin{array}{c}14 \\
(2.3 \%)\end{array}$ & 1.714 & 0.19 \\
\hline
\end{tabular}

Por el contrario, en relación a los candidatos con un perfil de habilidades medio, no se puede determinar que los responsables de selección de personal discriminan entre candidatos con antecedentes y sin antecedentes de prisión: la diferencia en la proporción de llamada no es lo suficientemente elevada como para poder descartar que esta diferencia se deba al azar $(P$-valor $>0,05)$. Se respondió sólo a los CV sin la marca del antecedente penal en el $2.3 \%$ de los casos, mientras que se obtuvo una respuesta positiva sólo en los currículos con la marca en el $1.2 \%$ de los casos.

Es posible que la incapacidad de detectar una diferencia significativa en los candidatos de perfil medio sea debido a la baja tasa de respuesta general para este perfil en un contexto de crisis económica. La falta de una diferencia relativa significativa entre estas dos probabilidades podría ser debida a que las diferencias en términos absolutos son muy pequeñas. Por este motivo, sospechamos que la potencia estadística de nuestro experimento no permite observar diferencias de esta magnitud ${ }^{13}$. En este sentido, es importante destacar que el trabajo de campo se realizó en uno de los peores momentos de la crisis económica española. Es probable que en un contexto de mayor contratación la tasa de respuesta para los candidatos hubiera sido más elevada y, por lo tanto, se hubieran podido observar diferencias significativas también para este perfil.

Este elemento conduce a reflexionar sobre la baja tasa de respuesta en el perfil de habilidades medio. Para estos casos, incluso sin mostrar la señal del paso por el sistema penal, los candidatos reciben una respuesta en menos del 5\% de los casos. Es preciso

\footnotetext{
${ }^{13}$ El tamaño de la muestra era adecuado para una probabilidad de error de tipo $1(\alpha)$ igual a 0.05 , un poder estadístico (1- $\beta$ ) igual a 0.80 , y una proporción de celdas discordantes de ambos tipos del $5 \%$ según el método señalado por Vuolo, Uggen y Lageson (2016).
} 
recordar que este perfil se correspondía al perfil de presos excarcelado "tipo" que se observaba en la investigación de Alós-Moner et al. (2011). Este dato ilustra las dificultades de un excarcelado "tipo" para encontrar empleo, independientemente del efecto del estigma, y consecuentemente, de las dificultades en las que se encuentran los programas de inserción laboral para internos con estas características.

\section{Discusión}

Los resultados de este estudio señalan que ciertas personas con antecedentes penales reciben un trato desfavorable en el mercado de trabajo en España y, por lo tanto, confirman la necesidad de considerar el impacto del efecto del estigma para entender la relación entre el paso por el sistema penal y la precariedad laboral al menos para algunas de las personas con una condena. A diferencia de investigaciones previas, la metodología experimental de los Correspondence Tests nos ha permitido aislar el efecto del estigma de otras posibles explicaciones (Bertrand \& Mullainathan, 2004; Pager, 2007).

Es importante realizar tres matizaciones a estos resultados. En primer lugar, se podría aducir que este experimento sólo permite establecer que existe el efecto del estigma para las personas con una señal de su paso por prisión. Sin embargo, la literatura en otros países señala que se puede esperar que, en menor medida, también exista un tratamiento desfavorable a condenados a cualquier tipo de pena y que, por tanto, podamos hablar de un efecto "antecedentes penales" en España (por ejemplo, Lott, 1990; Pager, 2007). En este sentido, Uggen, Vuolo, Lageson, Ruhland y WhitMan (2014) realizaron un experimento similar en Estados Unidos, pero, en vez de testar el efecto del paso por prisión, lo hicieron en relación a haber cometido una falta (misdemenour) por "alteración del orden público" (disordely conduct). Estos autores encontraron que mostrar la señal de un acto de este tipo también reducía la probabilidad de ser contactado para proseguir un proceso de selección de personal.

En segundo lugar, como se ha señalado con anterioridad, es preciso indicar que estos datos no reflejan el grado de discriminación de las personas con antecedentes penales en el mercado laboral (Heckman, 1998). La metodología del Correspondence Tests sólo permite determinar si los responsables de selección de personal tratan desfavorablemente a las personas que muestran una señal del paso por el sistema penal en su currículo y cuando no conocen al empleador, no el grado de discriminación real 
total. Sería interesante desarrollar en el futuro investigaciones con este objetivo mediante bases de datos administrativas o estudios longitudinales.

En tercer lugar, la investigación se ha centrado sólo en hombres jóvenes. Sin embargo, como muestran estudios realizados en otros países, el impacto de los antecedentes penales podría ser diferente en colectivos de más edad (Bernburg \& Krohn, 2003) y, sobretodo, en mujeres (Decker et al., 2015; Galgano, 2009; Verbruggen, 2016). Asimismo, durante la investigación se han omitido los problemas que generan los antecedentes penales a las personas inmigrantes (en este sentido ver Larrauri, 2016). Por lo tanto, para completar el diagnóstico de la influencia de los antecedentes penales en el conjunto de la población española, sería preciso desarrollar más estudios respecto a estos otros colectivos. Este trabajo sólo pretende ser un primer paso que ofrece una nueva estrategia en este camino.

Con el objetivo de completar el estudio del efecto del estigma, se está desarrollando nueva investigación para analizar los motivos que explican el comportamiento de los responsables de selección de personal (ver Rovira 2016; Rodríguez-Menés \& Rovira, 2018). El hecho de haber enviado CV según nivel de habilidades nos permite estudiar los mecanismos descritos por las teorías económicas de la discriminación. Desde estas teorías se establece que los responsables de selección de personal tratan desfavorablemente a las personas con un estigma por tres motivos distintos. En primer lugar, a causa de una animadversión de carácter moral -teorías de la “discriminación por animadversión”, ver Becker (1957)-. En segundo lugar, porque se utiliza la señal del estigma para inferir el nivel de la productividad laboral futura de los candidatos -teorías de la “discriminación estadística de primer orden”, ver Phelps (1972) y Spence (1973)-. En tercer lugar, la señal del estigma también podría provocar en los responsables de selección de personal una incertidumbre diferente, mayores o menores dudas, sobre las características de estas personas -teorías de la "discriminación estadística de segundo orden”, ver Heckman (1998)-. Para poder separar el efecto de cada uno de estos diferentes mecanismos en la actualidad se ha desarrollado una innovación metodológica. Los primeros resultados apuntan a que existe un comportamiento discriminador independientemente del nivel de habilidades de los candidatos en línea de lo establecido por las teorías de "discriminación por animadversión” aunque se advierte que en algunos empleos el hecho de mostrar los antecedentes penales puede ser incluso 
beneficioso (en línea de lo establecido por las teorías de la “discriminación estadística de segundo orden").

Desde un punto de vista práctico, este estudio señala que se debe tener en cuenta el efecto del estigma en las políticas públicas dirigidas a la reinserción laboral de las personas condenadas. Si bien es necesario continuar abordando los déficits de estas personas y tener en cuenta las consecuencias para el acceso al empleo derivadas del cumplimiento de la pena, esta investigación demuestra que también es necesario considerar que las personas con antecedentes penales son discriminadas en el mercado laboral por el hecho de haber sido condenadas.

Indirectamente, esta investigación también ilustra sobre las dificultades de los programas de inserción laboral dirigidos a este colectivo. Para el nivel de habilidades "medio", que recoge las características del ex-preso "tipo", la probabilidad de respuesta para poder acudir tan sólo a la entrevista laboral es inferior a un 5\% incluso para los candidatos sin la mención de los antecedentes. En este sentido, ante la valoración de la baja tasa de éxito de algunos programas de inserción, se debe ser consciente de que estos datos no significan necesariamente que estos programas sean innecesarios o que estén mal diseñados, sino que el contexto externo es tan negativo, que su campo de acción es muy limitado.

Sin embargo, esta situación ya inicialmente tan negativa, no debe hacernos olvidar la importancia del efecto del estigma. Si las personas con una baja formación y experiencia laboral ya tienen escasas perspectivas para insertarse en el mercado laboral, se debe evitar añadir obstáculos a su camino. Puede ser que precisamente en estas personas, con unas perspectivas laborales muy limitadas, el estigma les termine de cerrar las puertas existentes o les desanime para seguir intentándolo.

En conclusión, en España en algunos casos existe un trato desfavorable hacia las personas con antecedentes penales en el mundo laboral, cuando se advierte su condición. Por lo tanto, el estigma de la condena actúa como una "pena añadida" que va más allá de las obligaciones establecidas en la sentencia. Es urgente visibilizar este fenómeno e incorporarlo de forma decidida en las políticas de reinserción dirigidas a abordar esta problemática. En la medida en que el empleo parece ser un factor importante para los procesos de desistimiento del delito, el estigma de los antecedentes penales puede estar reforzando el círculo vicioso entre el paso por el sistema penal y la delincuencia. 


\section{Referencias}

Alós-Moner, R., Esteban, F., Jodar, P., Miguélez, F., Alcaide, V., \& López-Roldan, P. (2011). La inserció laboral dels exinterns dels centres penitenciaris de Catalunya. Barcelona: Centre d'Estudis Jurídics i Formació Especialitzada (CEJFE).

Apel, R., \& Sweeten, G. (2010). The Impact of Incarceration on Employment during the Transition to Adulthood. Social Problems, 57(3), 448-479. doi: 10.1525/sp.2010.57.3.448

Backman, C. (2012). Criminal Records in Sweden. Regulation of Access to Criminal Records and the Use of Criminal Background Checks by Employers. [Tesis doctoral] Göteborg: Department of Sociology; Sociologiska institutionen.

Becker, G. S. (1957). The Economics of discrimination. Chicago: University of Chicago Press.

Bernburg, J. G., \& Krohn, M. G. (2003). Labeling, life chances, and adult crime: The direct and indirect effects of official intervention in adolescence on crime in early adulthood. Criminology, 41(4), 1287-1318. doi: 10.1111/j.17459125.2003.tb01020.x

Bertrand, M., \& Mullainathan, S. (2004). Are Emily and Greg More Employable than Lakisha and Jamal? A Field Experiment on Labor Market Discrimination. American Economic Review, 94(4), 991-1013.

Bueno Arús, F. (2006). La Cancelación de antecedentes penales. Cizur Menor: Thomson Civitas.

Bushway, S. (1998). The Impact of an Arrest on the Job Stability of Young White American Men. Journal of Research in Crime and Delinquency, 35(4), 454-479. doi: $10.1177 / 0022427898035004005$

Cesaroni, C., \& Doob, A. N. (2003). The Decline in Support for Penal Welfarism. Evidence of Support among the Elite for Punitive Segregation. British Journal of Criminology, 43(2), 434-441. doi: 10.1093/bjc/43.2.434

Cid, J., \& Martí, J. (2012). Turning Points and Returning Points: Understanding the Role of Family Ties in the Process of Desistance. European Journal of Criminology, 9(6), 603-620. doi: 10.1177/1477370812453102

Clear, T. R. (2008). The Effects of High Imprisonment Rates on Communities. Crime and Justice, 37(1), 97-132. doi: 10.1086/522360

Crutchfield, R. D. (2014). Get a job. Labor Markets, Economic Opportunity and Crime. New York: New York University Press.

Decker, S. H., Ortiz, N., Spohn, C., \& Hedberg, E. (2015). Criminal stigma, race, and ethnicity: The consequences of imprisonment for employment. Journal of Criminal Justice, 43(2), 108-121. doi: 10.1016/j.jcrimjus.2015.02.002

Demleitner, N. V. (1999). Preventing internal exile: the need for restrictions on collateral sentencing consequences. Standford Law \& Policy Review, 11, 153-171.

Denver, M., Siwach, G., \& Bushway, S. D. (2017). A new look at the employment and recidivism relationship through the lens of a criminal background check. Criminology, 55(1), 174-204. doi: 10.1111/1745-9125.12130

Departament de Justícia. Generalitat de Cataluña. (2015a). Descriptors Estadístics Serveis Penitenciaris: 6.3.2 Dones. Altes des de llibertat. Històric.

Departament de Justícia. Generalitat de Cataluña. (2015b). Descriptors estadístics serveis penitenciaris. 2.1 Temps d'estada a presó.

Díez Ripollés, J. L. (2004). El nuevo modelo penal de la seguridad ciudadana. Revista 
Electrónica de Ciencia Penal y Criminología, (6), 1-34.

Galgano, S. W. (2009). Barriers to Reintegration: An Audit Study of the Impact of Race and Offender Status on Employment Opportunities for Women. Social Thought and Research, 30, 21-37.

Garland, D. (2001). The Culture of Control: Crime and Social Order in Contemporary Society. Chicago: University of Chicago Press.

Goffman, E. (1990). Stigma: notes on the management of spoiled identity. UK: Penguin Psychology.

Granovetter, M. (1995). Getting a job: a study of contacts and careers. Chicago: The University of Chicago Press.

Grogger, J. (1995). The Effect of Arrests on the Employment and Earnings of Young Men. The Quarterly Journal of Economics, 110(1), 51-71. doi: 10.2307/2118510

Grosso Galván, M. (1984). Los Antecedentes Penales: Rehabilitación y Control Social. Barcelona: Editorial Bosch.

Gutiérrez-Maldonado, J., \& Sintas, F. (1995). Actitud de los Empresarios hacia la Integración Laboral de Personas que están o han estado Sometidas a Medidas de Privación de Libertad. Anuario de Psicología Jurídica, 5(3), 18-45.

Harding, D. J. (2003). Jean Valjean's dilemma: the management of ex-convict identity in the search for employment. Deviant Behavior, 24(6), 571-595. doi: $10.1080 / 713840275$

Heckman, J. J. (1998). Detecting Discrimination. Journal of Economic Perspectives, 12(2), 101-116.

Holzer, H. J., Raphael, S., \& Stoll, M. A. (2007). The Effect of an Applicant's Criminal History on Employer Hiring Decisions and Screening Practices: Evidence from Los Angeles. En S. Bushway, M. A. Stoll, \& D. F. Weiman (Eds.), Barriers to Reentry? The Labor Market for Released Prisoners in Post-Industrial America (pp. 117-150). New York: Russell Sage Foundation.

Instituto Nacional de Estadística. (2013). Datos procedentes de la Estadística de Nacimientos.

Instituto Nacional de Estadística. (2015). Tasas de paro por distintos grupos de edad, sexo y comunidad autónoma.

Larrauri, E. (2016). Antecedentes penales y expulsión de personas inmigrantes. InDret, $2 / 2016$.

Larrauri, E., \& Jacobs, J. B. (2011). Reinserción laboral y antecedentes penales. Revista Electrónica de Ciencia Penal y Criminología, 13(9), 1-25.

LeBel, T. P. (2008). Perceptions of and Responses to Stigma. Sociology Compass, 2(2), 409-432. doi: 10.1111/j.1751-9020.2007.00081.x

Loeffler, C. E. (2013). Does Imprisonment Alter the Life Course? Evidence on Crime and Employment From a Natural Experiment. Criminology, 51(1), 137-166. doi: $10.1111 / 1745-9125.12000$

Lott, J. R. (1990). The Effect of Conviction on the Legitimate Income of Criminals. Economics Letters, 34, 381-385. doi: 10.1016/0165-1765(90)90149-U

Maruna, S. (2011). Judicial Rehabilitation and the "Clean Bill of Health" in Criminal Justice. European Journal of Probation, 3(1), 97-117.

Mauer, M., \& Chesney Lind, M. (2003). Invisible Punishment: The Collateral Consequences of Mass Imprisonmen. New York: The New Press.

Merton, R. K. (1938). Social Structure and Anomie. American Sociological Review, 3(5), 672-682. 
Metcalf, H., Anderson, T., \& Rolfe, H. (2001). Barriers to Employment for Offenders and Ex-offenders. Part One. Research Report - Department of Work and Pensions. Research Report No 155. London: Corporate Document Services.

Miguélez, F., Alós-Moner, R., Martín, A., \& Gibert, F. (2006). El treball a les presons de Catalunya. Barcelona: Centre d'Estudis Jurídics i Formació Especialitzada (CEJFE).

Nagin, D., \& Waldfogel, J. (1995). The Effects of Criminality and Conviction on the Labor Market Status of Young British Offenders. International Review of Law and Economics, 15(1), 109-126.

Pager, D. (2007). Marked: Race, Crime, and Finding Work in an Era of Mass Incarceration. Chicago: University of Chicago Press.

Phelps, E. S. (1972). The Statistical theory of Racism and Sexism. American Economic Review, 62(4), 659-661. doi: 10.2307/1806107

Ramakers, A., van Wilsem, J., \& Apel, R. (2012). The effect of labour market absence on finding employment: A comparison between ex-prisoners and unemployed future prisoners. European Journal of Criminology, 9(4), 442-461. doi: $10.1177 / 1477370812448278$

Real Academia Española. (2016a). Antecedente. En Diccionario de la lengua española Madrid: Real Academia Española.

Real Academia Española. (2016b). Discriminar. En Diccionario de la lengua española. Madrid: Real Academia Española.

Riach, P. A., \& Rich, J. (2004). Deceptive Field Experiments of Discrimination: Are They Ethical? Kyklos, 57(3), 457-470. doi: 10.1111/j.0023-5962.2004.00262.x

Rieucau, G. (2008). Job advertisements and personal networks: two specific channels in the Spanish labour market. Transfer: European Review of Labour and Research, 14(3), 469-480.

Ríos, J. C., \& Cabrera, P. J. (1998). Mil voces presas. Madrid: Universidad Pontificia.

Rodríguez-Menés, J., \& Rovira, M. (2018). [Aceptado condicionalmente] Assessing discrimination in correspondence studies. Sociological Methods and Research.

Roldán, H. (2010). El uso de la libertad condicional y su influencia en el tamaño de la población reclusa en España. Revista Electrónica de Ciencia Penal y Criminología, 12(4), 1-17.

Rovira, M. (2016). Antecedentes penales y mercado laboral. [Tesis doctoral] Universidad Pompeu Fabra.

Sampson, R. J., \& Laub, J. H. (1993). Crime in the Making :Pathways and Turning Points Through Life. Cambridge: Harvard University Press.

Spence, M. (1973). Job Market Signaling. The Quarterly Journal of Economics, 87(3), 355-374. doi: 10.2307/1882010

Sutherland, E., \& Cressey, D. (1978). Criminology (10th ed.). Philadelphia: JB Lippincott.

Thomas, T. (2007). Criminal Records A Database for the Criminal Justice System and Beyond. Houndmill: Palgrave Macmillan.

Uggen, C., Manza, J., \& Thompson, M. (2006). Citizenship, Democracy, and the Civic Reintegration of Criminal Offenders. The ANNALS of the American Academy of Political and Social Science, 605(1), 281-310. doi: 10.1177/0002716206286898

Uggen, C., \& Staff, J. (2001). Work as a Turning Point for Criminal Offenders. Corrections Management Quarterly, 5, 1-16. doi: 10.1080/07418825.2014.894111

Uggen, C., Vuolo, M., Lageson, S., Ruhland, E., \& WhitMan, H. (2014). The edge of 
stigma: An experimental audit of the effects of low-level criminal records on employment. Criminology, 52(4), 627-654. doi: 10.1111/1745-9125.12051

van der Geest, V. R., Bijleveld, C. C. J. H., Blokland, A. A. J., \& Nagin, D. S. (2016). The Effects of Incarceration on Longitudinal Trajectories of Employment: A Follow-Up in High-Risk Youth From Ages 23 to 32. Crime \& Delinquency, 62(1), 107-140. doi: 10.1177/0011128713519196

Verbruggen, J. (2016). Effects of Unemployment, Conviction and Incarceration on Employment: A Longitudinal Study on the Employment Prospects of Disadvantaged Youths. British Journal of Criminology, 56(4), 729-749. doi: 10.1093/bjc/azv074

Visher, C. A., \& Kachnowsi, V. (2007). Finding Work on the Outside: Results from the "Returning Home" Project in Chicago. En Barriers to Reentry? The Labor Market for Released Prisoners in Post-Industrial America (pp. 80-113). New York: Russell Sage Foundation.

Vuolo, M., Uggen, C., \& Lageson, S. (2016). Statistical Power in Experimental Audit Studies: Cautions and Calculations for Matched Tests With Nominal Outcomes. Sociological Methods \& Research, 45(2), 260-303. doi: $10.1177 / 0049124115570066$

Western, B. (2002). The Impact of Incarceration on Wage Mobility and Inequality. American Sociological Review, 67(4), 526-546. doi: 10.2307/3088944

\section{Agradecimientos}

Quiero agradecer a mis directores de tesis la Dra. Elena Larrauri y el Dr. Jorge Rodriguez por su atento apoyo en el desarrollo de este trabajo. También a los miembros de mi tribunal de tesis doctoral el Dr. José Luís Díez Ripollés, la Dra. Esther Fernández Molina y el Dr. Diego Torrente, y a los dos revisores anónimos de la revista, por sus incisivos comentarios que si duda han ayudado a mejorar esta versión. También debo agradecer a Assumpta Sopeña, Ana Yael Zareceansky y Adrià Giol su colaboración en diferentes aspectos del trabajo. Los errores son exclusivamente responsabilidad mía.

\section{Financiación}

Este trabajo se ha enmarcado en los proyectos "La regulación de los antecedentes penales: su efecto en el acceso al mercado laboral de los jóvenes", financiado por la convocatoria de ayudas a la investigación RecerCaixa (Ref. RecerCaixa 2013), "Ejecución y supervisión de la pena: Calidad de la intervención, legitimidad y reincidencia", financiado por el Ministerio de Economía y Competitividad (MINECO) (Ref. DER2015-64403-P y FEDER, UE); y el proyecto "Penología europea: Su influencia en el sistema de penas español", financiado por la 'Agència de Gestió d'Ajuts Universitaris i de Recerca' de la Generalitat de Catalunya (Ref. DURSI-AGAUR SGR2014 426).

Martí Rovira es Doctor en Derecho (Criminología) por la Universidad Pompeu Fabra (UPF). Su tesis doctoral "Antecedentes penales y mundo laboral", fue dirigida por Elena Larrauri y Jorge Rodríguez-Menés. Previamente, Martí Rovira cursó la licenciatura en 
Ciencias Políticas y de la Administración en la UPF, el máster en Técnicas de Investigación Social Aplicada en la Universitat Autònoma de Barcelona (UAB) y el MSc en "Sociology of Crime, Control and Globalisation" en la London School of Economics and Political Science (LSE). Actualmente, su investigación se centra en la calidad de vida en prisión y las consecuencias colaterales de las condenas. 